\title{
Solar Wind and Heliospheric Compositional Variations
}

\author{
A. B. Galvin
}

Institute for the Study of Earth, Oceans, and Space and the Department of Physics, University of New Hampshire, Durham, NH, 03824 USA

\begin{abstract}
Recent in-situ measurements of the composition of the solar wind and heliospheric particles from SOHO, ACE, Wind and Ulysses are providing valuable and often unique new information on origins and acceleration processes in the Sun and heliosphere. This paper reviews some of the more interesting recent findings and their implications.
\end{abstract}

\section{Introduction}

It has long been recognized that solar wind relative abundances remain essentially true to whatever composition was established as the solar wind accelerated out of the corona and can therefore be used to learn about the physical conditions and processes at the solar source region (Hundhausen et al. 1968). Further, compositional and spectral signatures of suprathermal and energetic heliospheric particle populations (e.g., pickup ions, SEP, CIR events) provide valuable information on these ions' source populations, injection and acceleration processes, and transport effects.

Recent solar wind and heliospheric studies have benefited from a variety of new or improved composition measurement techniques, including the use of socalled "time-of-flight" spectrometers. These include solar wind and suprathermal composition experiments on Ulysses, Wind, ACE, and SOHO (Gloeckler et al. 1992, 1995 \& 1998; Hovestadt et al. 1995). A significant advancement in sensitivity and resolution for energetic ion charge states has recently been accomplished on ACE (Moebius et al. 1998).

\section{Solar Wind Ion Composition}

There are compositional (elemental and charge state) signatures that are specifically related to solar wind flow type (coronal hole streams, interplanetary coronal mass ejections, or slow solar wind) as discussed by von Steiger et al. $(1995,2000)$ and Galvin (1997). For example, the abundances of the elements measured in the corona and in the solar wind are different from photospheric values. The differences are mostly determined by the so-called FIP effect (first ionization potential). The process somehow separates neutral and ionized particles in the chromosphere, resulting in a fractionated sample of solar material passing into the corona, out of which the solar wind is accelerated. The underlying physical process responsible for the FIP effect is not exactly known, although many scenarios can reasonably reproduce the coronal abundances (Henoux 1998). Recent 
observations with Ulysses/SWICS indicate that the strength of the fractionation may not be as strong as previously reported, with the slow solar wind showing an average enrichment factor of 3 (rather than the canonical value of 4 ) and the fast solar wind with an enrichment factor only about half that of the slow solar wind (von Steiger et al. 2000). Recent observations of magnetic cloud elemental composition by SOHO/CELIAS indicate that these types of solar wind show additional mass fractionation over slow solar wind values (Wurz et al. 2000).

\subsection{ICME Solar Wind Composition}

The interplanetary manifestations of coronal mass ejections (ICMEs) exhibit tremendous variability in their internal structure and composition. As noted in the review by Galvin (1997), solar wind with very high charge states are indictative of ICMEs. However, the converse is not true. ICMEs exhibit a broad range of charge state (and elemental) composition, varying from event to event and even within a given event. Often, the highest charge states (e.g., Fe $z$ 16) are associated with the ICME subclass known as magnetic clouds (Henke et al. 1998). Another type of ICME, observed at high latitudes by Ulysses during its first solar polar orbit, exhibits charge state composition (e.g., Fe 10-11) similar to the polar hole solar wind (Galvin 1997; Galvin \& Gloeckler, 1997). These extremes bracket the "in between" ICME compositions (see Figure 7 of Galvin \& Gloeckler). Even when the ICME is characterized by exceptionally high charge states, lower nominal charge states are typically present (Galvin 1997 and references therein).

In some cases, ICME solar wind is exceptional for its anomalously low charge states (Galvin 1997 and references therein; Ho et al. 2000; Burlaga et al. 1998; Gloeckler et al. 1999; Skoug et al. 1999). Here there are compositional features that are not observed in the solar wind under normal circumstances, including the presence of solar wind $\mathrm{He}^{+}$, and minor ion charge states such as $\mathrm{C}^{+2}$, $\mathrm{O}^{+2,+3}, \mathrm{Fe}^{+3}$. These charge states indicate formation temperatures on the order of $10^{5} \mathrm{~K}$, more indicative of prominence material than of coronal origins. These extremely low charge states are sometimes isolated within the associated ICME event, as seen in the January 11, 1997 event reported by Burlaga et al. (1998). In other cases, these low charge states co-exist with higher charge states, as seen in the May 1998 event (Gloeckler et al. 1999; Popecki et al. 2000). The mixture charge-state distributions may be indicative of extreme non-equilibrium conditions in closed magnetic structures, in which rapid expansion cools the plasma much faster than the recombination process (Neukomm \& Bochsler 1996). Another interesting feature of these events is that they have in some instances been associated with an enhanced abundance in the solar wind isotope ${ }^{3} \mathrm{He}$ (Ho et al. 2000).

ICMEs seem to permit strong mass fractionation, as reported by Wurz et al. (1998) in the January 1997 ICME event (Figure 1a). This event began with a CME launch on January 6, which arrived as an ICME at SOHO on January 10. The ICME has been characterized as a magnetic cloud followed on January 11 by a high density "plug" of filamentary (solar prominence) material (Burlaga et al. 1998). Wurz et al. observe a monotonic enhancement of heavy elements as a function of mass for both the magnetic cloud and filamentary material when compared to slow solar wind. Enrichment factors of up to 4 are seen for Si/O and 

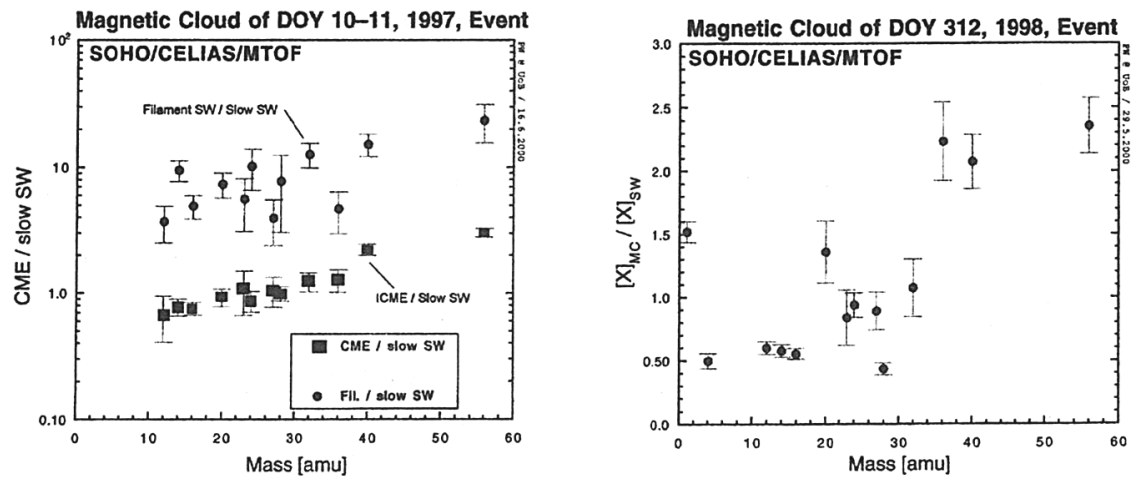

Figure 1. Solar wind elemental composition of ICMEs relative to slow solar wind (courtesy P. Wurz). (a) Magnetic cloud material and filamentary material observed during the January 10-11, 1997 ICME event. (b) Magnetic cloud material during the November 8, $1998 \mathrm{ICME}$ period.

$\mathrm{Fe} / \mathrm{O}$ over the reference slow solar wind. Similar results have been seen for other magnetic cloud events in 1997-1998 (e.g., Figure 1b), as reported by Wurz et al. (2000). Wurz, Bochsler \& Lee (2000) present a theoretical model which can reproduce the observations. Their model assumes hot coronal loops with nonMaxwellian electron distributions as the precursor to the CME. Perpendicular diffusion across the magnetic field lines results in the preferential loss of lighter ions from the loop, leading to the observed mass fractionation.

\section{Heliospheric Composition}

Composition plays an important role in distinguishing source populations and in tracing the injection and acceleration processes for suprathermal and energetic particles in the heliosphere.

\subsection{Pickup Ions}

Neutral interstellar gas is free to penetrate the magnetic barrier of the heliosphere. A portion of these neutrals becomes ionized - principally by photoionization (solar EUV radiation), charge exchange with the solar wind, and/or electron collisional ionization. These freshly created ions are "picked up" and transported in the solar wind, having speeds up to twice that of the solar wind. The first observations of interstellar pickup ions was of $\mathrm{He}^{+}$(Moebius et al. 1985). Ulysses SWICS has detected pickup ions of interstellar $\mathrm{H}, \mathrm{N}, \mathrm{O}$, and $\mathrm{Ne}$ origin (Gloeckler et al. 1993; Geiss et al. 1994). These pickup ions provide a tool for studying the interstellar gas and its interaction with the heliosphere.

Recent $\mathrm{He}^{+}$pickup ion measurements by SOHO CELIAS provide direct evidence of the interstellar helium gas flow velocity at 1 AU (Moebius et al. 1999a). CELIAS measures the parameters for determining ionization rates (solar EUV flux, the solar wind speed and density) as well as the pickup ion distribution 
itself. Observations taken while the spacecraft was on the upwind side of the interstellar gas with respect to the Sun (June-July 1996) indicate the cut-off speed significantly exceeds the nominal value of $2^{*} \mathrm{Vsw}$. Moebius et al. note that adding the speed gained through gravitational attraction to the initial local interstellar medium's flow speed $(25 \mathrm{~km} / \mathrm{s})$ would lead to speeds $\gtrsim 50 \mathrm{~km} / \mathrm{s}$ at $1 \mathrm{AU}$.

Pickup ions $\left(\mathrm{C}^{+}\right)$from an inner heliospheric source were first reported by Geiss et al. (1995), who suggested these pickup ions result from the release of neutrals from dust grains, which then undergo the ionization and pickup process. Gloeckler et al. (2000) have performed a detailed analysis of the Ulysses low and middle latitude data within $2 \mathrm{AU}$. They find sufficient similarities between the relative abundances of inner source $\mathrm{H}, \mathrm{C}, \mathrm{N}, \mathrm{O}, \mathrm{Ne}, \mathrm{Mg}$, and Si pickup ions and corresponding solar wind ions to suggest that the inner source is in fact recycled solar wind. Solar wind is implanted in the dust grains, released as slow moving neutrals, then become pickup ions. These observations indicate a rather close relationship between the dust and solar wind.

\subsection{Ion Acceleration at CME-Driven Shocks}

Gradual Solar Energetic Particle (SEP) events are associated with fast CMEs and type II radio bursts. The shock wave driven by a fast CME is the most likely origin of gradual SEP events, providing an acceleration site for various source populations. These potential sources include solar wind ions, pickup ions, and any residual particles in the vicinity from previous SEP events. New data from SOHO, Wind, and ACE provide important insights on determining the relative importance of different source populations and the associated mechanisms of injection and acceleration.

Enhanced $\mathrm{He}^{+}$abundances were frequently observed in gradual SEP events over twenty years ago (Hovestadt et al. 1981). At the time of these earlier studies, the existence of pickup ions had not been empirically established. Today, measurements of the $\mathrm{He}$ ionic charge composition in CME-related SEPs are possible in detail over an extended energy range. Figure $2 b$ shows the gradual SEP time period 10 December 1997 , with a $\mathrm{He}^{+} / \mathrm{He}^{+2}$ ratio $\sim 1$. The energy spectrum for $\mathrm{He}^{+}$shows a smooth transition from pickup ion energies $\left(\leqslant 2^{*} \mathrm{Vsw}\right)$ to suprathermal energies. This suggests that the source of the $\mathrm{He}^{+}$, at least in this event, is indeed pickup ions.

Event-averaged measurements of gradual SEP heavy ions $(\mathrm{Z}>2)$ have long established that their charge states are solar wind-like (Luhn et al. 1984). Interesting variations from this general result have now emerged with ACE. Klecker et al. (2000) have compared solar wind and gradual SEP elemental and charge state composition for $\mathrm{C}, \mathrm{O}$, and Fe ions. In the three gradual SEP events studied, they found that the mean ionic charge of $\mathrm{C}$ was comparable in the solar wind and the SEP particles $(\mathrm{C} \sim 5.3-5.5, \sim 5.5-5.6$ for solar wind and SEP, respectively), however a modest increase was seen in the SEP charge states for $\mathrm{O}$ and $\mathrm{Fe}(\mathrm{O} \sim 6.1-6.2$ vs. $\sim 6.7-6.8$ for solar wind vs. SEP; $\mathrm{Fe} \sim 9.6-10.1$ vs. $\sim$ 10.8-11.7 for solar wind vs. SEP). They attribute these differences to $M / Q$ -dependent acceleration affects at the accompanying interplanetary shock.

In another study combining solar wind and SEP data, Popecki et al. (2000) compared SEP Fe charge states with solar wind Fe during the magnetic cloud 

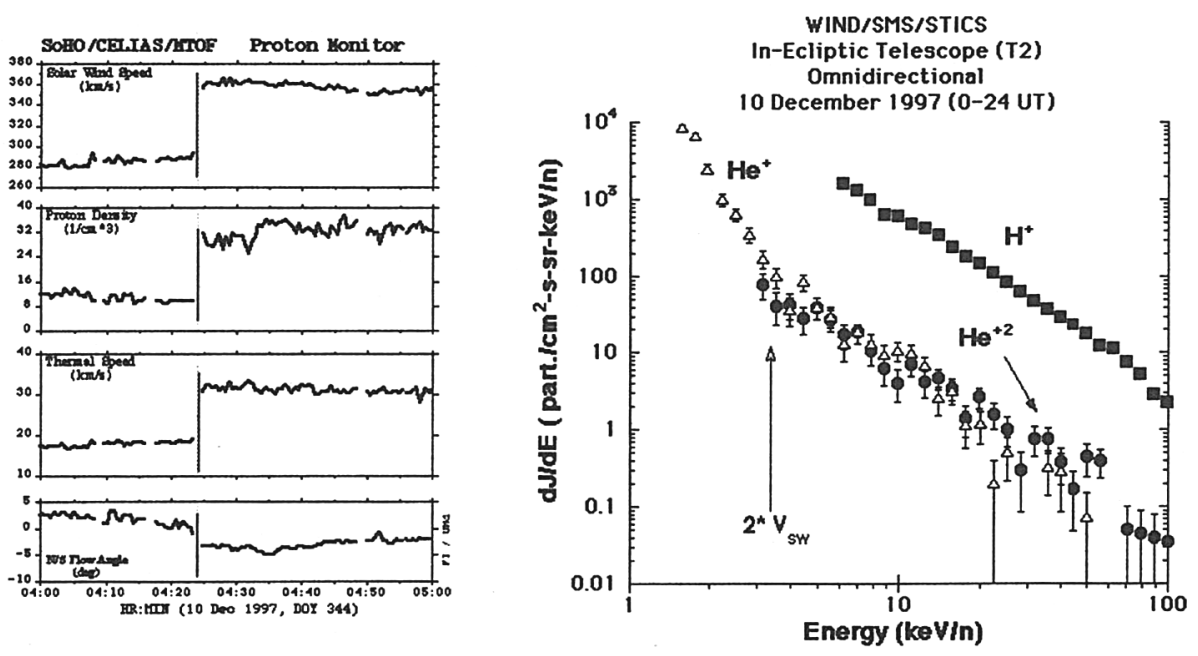

Figure 2. (a) Interplanetary shock observed on 10 December 1997 by the CELIAS Proton Monitor on SOHO (courtesy F.M. Ipavich). (b) Suprathermal $\mathrm{He}^{+}, \mathrm{He}^{+}$and $\mathrm{He}^{+2}$ measured by the Wind STICS sensor on December 10,1997 (0-24 UT). Ions are from the in-ecliptic telescope (T2), with full azimuthal coverage.

event of May 2-3, 1998. They observed similar temporal onsets for high $\mathrm{Fe}$ charge states $(Q \gtrsim 14)$, approximately associated with the arrival of the cloud. They suggest that the footpoints of the magnetic cloud remained connected near an active region on the Sun for 3 to 4 days. Wimmer-Schweingruber, Kern \& Hamilton (1999) also found compositional similarities for the ICME solar wind and SEP material in the November 1997 events, again suggesting a common source reservoir may be involved.

In some (not all) instances, gradual SEP events have shown a significant energy dependence in the mean ionic charge of heavy elements (Moebius et al. 1999b; Mazur et al. 1999). Bogdanov et al. (2000) have combined CELIAS and SEPICA data to extend gradual SEP O and Fe charge state measurements over the energy range 0.02 to $0.7 \mathrm{MeV} / \mathrm{n}$. They find large variations in the amount of energy dependence for $\mathrm{Fe}$ from event to event. For cases of local acceleration at the CME-related shock, the variation in SEP Fe charge states over the observed energy interval is on the order of 1-2 charge units, which they attributed to M/Qdependent affects. In other cases, the Fe mean charge state variation reached $\sim 4$ charge units. In these instances, they suggest the acceleration occurred in the dense coronal plasma, where stripping and recombination effects would be important.

\subsection{CIR Particle Events}

While solar activity allows one to observe the intriguing and varied compositional signatures of ICME and SEP events, solar minimum conditions provide an equally interesting opportunity to study the ion events associated with coro- 

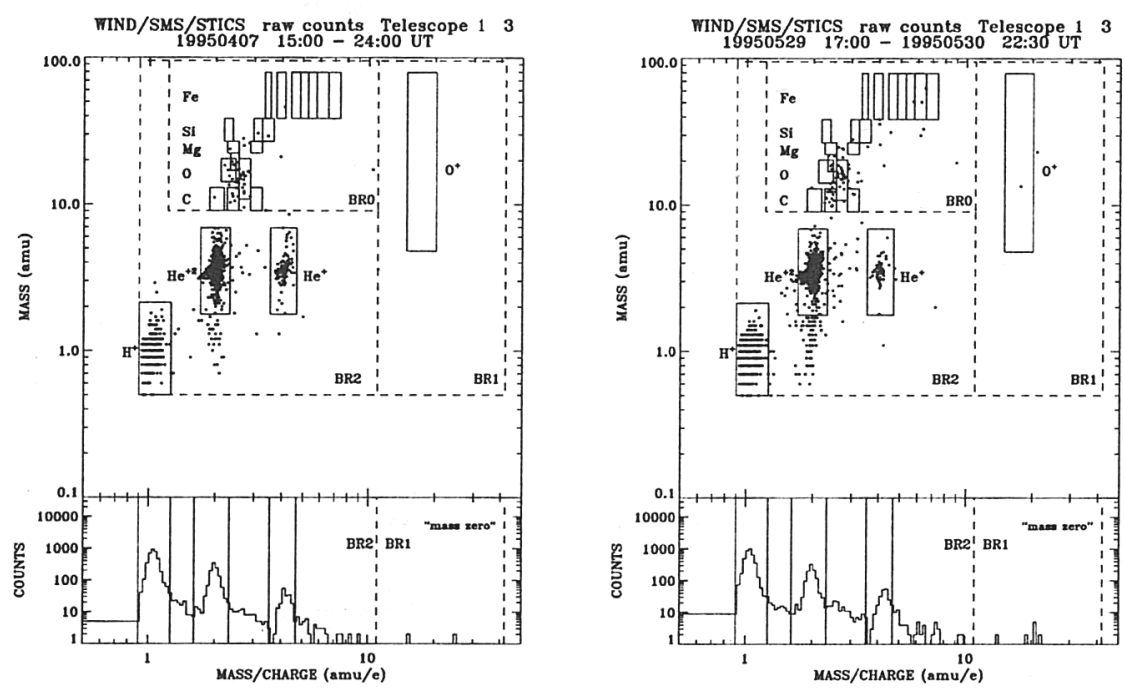

Figure 3. Suprathermal particles measured by the Wind STICS sensor near $1 \mathrm{AU}$ during two CIRs events. Ions are measured with full azimuthal coverage from the two out-of-ecliptic telescopes (T1, T3). The upper panels show scatter plots of ions for which both the mass and the $M / Q$ are determined. The bottom panels are count histograms for those ions in which only the $M / Q$ could be measured.

tating interaction regions (CIRs). CIRs are formed when fast solar wind from a coronal hole overtakes a slow solar wind, creating a compression region of both fast and slow solar wind. Typically at $>2 \mathrm{AU}$, a forward and reverse shock pair forms, becoming a site for particle acceleration. CIR particles are observed to have energies from 10's $\mathrm{keV} / \mathrm{n}$ up to several MeV/n (Mason \& Sanderson 1999). Some of these energetic ions propagate back to the inner heliosphere through magnetic field connection where they can be studied by near-Earth satellites.

The outer heliosphere may not be the only CIR acceleration site. Results from SOHO/CELIAS show efficient acceleration occurring in CIRs near $1 \mathrm{AU}$. $\mathrm{He}^{+} / \mathrm{He}^{+2}$ ratios of $\sim 0.16-0.31$ were observed for the energy range 25-150 keV/n (Hilchenbach et al. 1999). Spectral analysis of $\mathrm{H}^{+}, \mathrm{He}^{+}$, and $\mathrm{He}^{+2}$ ranging from solar wind speeds (Vsw) up to $>20 * \mathrm{Vsw}$ at $1 \mathrm{AU}$ have been reported by Chotoo et al. (2000). They suggest that the suprathermal CIR ions in the observed events originated within $0.5 \mathrm{AU}$. The source population appeared to be local suprathermal populations, such as the tail of the solar wind proton and alphas, and pickup $\mathrm{He}^{+}$. Their energy spectra showed a smooth transition from the CIR particles down to the solar wind for protons and alphas.

ACE SEPICA energetic ion observations reported by Morris et al. (2000) show the $\mathrm{He}^{+} / \mathrm{He}^{+2}$ ratio increases $\sim$ linearly with time after crossing the stream-stream interface within the CIR, indicating an increasing importance of interstellar pickup ions $\left(\mathrm{He}^{+}\right)$versus the solar wind $\left(\mathrm{He}^{+2}\right)$ for the source popu- 
lation as the observer is connected to the CIR from larger distances. Gloeckler et al. (2000) have suggested that the inner source of pickup ions may be a significant contributor to the heavy ions $(\mathrm{C}, \mathrm{N}, \mathrm{O}, \mathrm{Ne}, \mathrm{Mg}, \mathrm{Si})$ in the CIR population. If this is the case, then the accelerated particles should exhibit a significant fraction of low charge states. Figure 3 shows suprathermal ions observed by Wind STICS encompassing the same two CIR time periods of Chotoo et al. (2000). In addition to the previously reported $\mathrm{H}^{+}, \mathrm{He}^{+1,+2}$, one sees solar wind-like charge states for oxygen and carbon $\left(\mathrm{O}^{+6,+7}, \mathrm{C}^{+5}\right)$. There are a few counts for $M / Q>10$; both periods have a few counts near $M / Q \sim 16$, and the second period also at $\mathrm{M} / \mathrm{Q} \sim 20$, the nominal locations for $\mathrm{O}^{+}$and $\mathrm{Ne}^{+}$, respectively. Initial results from ACE SEPICA indicate the majority of CIR heavy ion (C, $\mathrm{O}, \mathrm{Ne}, \mathrm{Mg}$ ) charge states at $\sim 500 \mathrm{keV} / \mathrm{n}$ are similar to solar wind charge state distributions, indicating the solar wind (or its suprathermal tail) as the primary source (Moebius 2000). SEPICA measures a small contribution from low $\mathrm{Ne}$ charge states $(Q \sim 1,<8 \%)$. Both helium and neon can represent interstellar as well as inner source pickup ions at $1 \mathrm{AU}$. Obviously, there is more to be learned regarding potential source populations in CIR events.

\section{The Future}

The current complementary composition experiments on Ulysses, SOHO, Wind and ACE are enabling us for the first time to study in detail the solar wind and suprathermal heliospheric ions within the context of acceleration and fractionation processes both on the Sun and in interplanetary space. The role of various source populations for CIR and gradual SEP events are beginning to be ascertained.

Future missions with exceptional composition opportunities are currently planned. Genesis (expected launch 2001) will expose foils in various solar wind regimes and return those samples to Earth for detailed elemental and isotopic analysis. STEREO (expected launch late 2004) will be a two spacecraft mission, with one spacecraft leading and the other trailing in Earth's orbit. Both spacecraft will have identical payloads, including in-situ solar wind and energetic composition experiments and remote imaging experiments. It will be possible to directly relate in-situ composition obtained by one spacecraft with the limb images of the originating solar structure observed by the other spacecraft.

Acknowledgments. This paper was funded under NASA grants NAG56912 and NAG5-8044. A portion of this work was performed while a guest of the Max Planck Institute for Extraterrestrial Physics (Germany) during the summer of 1999.

\section{References}

Bogdanov, A., et al. 2000, in Acceleration and Transport of Energetic Particles Observed in the Heliosphere: ACE 2000 Symp., ed. Mewaldt et al., 143

Burlaga, L.F., et al. 1998, J. Geophys. Res., 103, 277

Chotoo, K., et al. 2000, J. Geophys. Res., 105, 23107 
Galvin, A.B. 1997, in Coronal Mass Ejections, Geophys. Monograph 99, ed. Crooker, Joselyn \& Feynman (Washington D.C.), 253

Galvin, A.B. \& Gloeckler, G. 1997, in Correlated Phenomena at the Sun, in the Heliosphere and in Geospace, ESA SP-415, 323

Geiss, J., et al. 1994, Astron. Astrophys., 282, 924

Geiss, J., et al. 1995, J. Geophys. Res., 100, 23373

Gloeckler, G., et al. 1992, Astron. Astrophys. Suppl. Ser., 92, 267

Gloeckler, G., et al. 1993, Science, 261, 70

Gloeckler, G., et al. 1995, Space Sci. Rev., 71, 79

Gloeckler, G., et al. 1998, Space Sci. Rev., 86, 497

Gloeckler, G., et al. 1999, Geophys. Res. Lett., 26, 157

Gloeckler, G., et al. 2000, J. Geophys. Res., 105, 7459

Henke, T., et al. 1998, Geophys. Res. Lett., 25, 3465

Henoux, J. 1998, Space Sci. Rev., 85, 215

Hilchenbach, M., et al. 1999, in Solar Wind Nine, ed. Habbal et al., 605

Ho, G., et al. 2000, Geophys. Res. Lett., 27, 309

Hovestadt, D., et al. 1981 ApJ, 246, L81

Hovestadt, D., et al. 1995, Sol. Phys., 162, 441

Hundhausen, A.J., Gilbert, H.E. \& Bame, S.J. 1968, J. Geophys. Res., 73, 5485

Klecker, B., et al. 2000, in Acceleration and Transport of Energetic Particles Observed in the Heliosphere: ACE 2000 Symp., ed. Mewaldt et al., 135

Luhn, A., et al., 1984, Adv. Space Res., 4, 161

Mason, G.M. \& Sanderson, T.R. 1999, Space Sci. Rev., 89, 77

Mazur, J.E., et al. 1999, Geophys. Res. Lett., 26, 173

Moebius, E. 2000, EOS Trans. (abstract), AGU, 81, SH72A-09, F974

Moebius, E. et al. 1985, Nature, 318, 426

Moebius, E., et al. 1998, Space Sci. Rev., 86, 449

Moebius, E., et al. 1999a, Geophys. Res. Lett., 26, 3181

Moebius, E., et al. 1999b, Geophys. Res. Lett., 26, 145

Morris, D., et al. 2000, COSPAR (abstract), Germany

Neukomm, R. \& Bochsler, P. 1996, ApJ, 465, 462

Popecki, M.A., et al. 2000, in Acceleration and Transport of Energetic Particles Observed in the Heliosphere: ACE 2000 Symp., ed. Mewaldt et al., 139

Skoug, R.M., et al. 1999, Geophys. Res. Lett., 26, 161

von Steiger, R., et al. 1995, Adv. Space Res., 15, 73

von Steiger, R., et al. 2000, J. Geophys. Res., 105, 27217

Wimmer-Schweingruber, R.F., Kern, O. \& Hamilton, D.C. 1999, Geophys. Res. Lett., 26, 3541

Wurz, P., et al. 1998, Geophys. Res. Lett., 25, 2557

Wurz, P., et al. 2000, EOS Trans. (abstract), AGU, 81, SH51B-10, F954

Wurz, P., Bochsler, P. \& Lee, M.A. 2000, J. Geophys. Res., 105, 27239 